\title{
In Vivo Visualization of Amyloid-like Structures in Humans
}

\author{
V. Kepe,* G.W. Small,** and J.R. Barrio* \\ * Department of Molecular and Medical Pharmacology \\ ** Department of Psychiatry and Biobehavioral Sciences, David Geffen School of Medicine, \\ University of California at Los Angeles, Los Angeles, CA 90095
}

Neurodegenerative diseases are progressive brain disorder ranging from dementias to movement disorders. Their pathological hallmarks include neuronal degeneration in disease-specific neuronal populations and disconnection of specific neuronal circuitry leading to specific symptoms as well as deposition of insoluble proteinaceous aggregates of different disease specific proteins intra- and/or extracellularly in the affected areas. Understanding of pathological molecular mechanisms of these diseases is critical for development of effective treatments and diagnostic methods which would allow detection in the earliest possible stage of disease progression, possibly at pre-clinical stages when the brain damage is still limited, are necessary for therapeutic interventions to be successful.

In contrast to the brain cells which have multiple targets suitable for in vivo molecular imaging detection (e.g. enzymes, neurotransmitter receptors) neuropathological protein aggregates are inert structures per se and their in vivo detection presents a considerable challenge.

Several types of radiolabeled molecular imaging probes with demonstrated capacity to bind to amyloid-like aggregates of some of these proteins in vitro have been developed over last two decades. Several of those have been radiolabeled with positron emitting isotopes and applied for in vivo brain positron emission tomography (PET) imaging of Alzheimer's disease and other neurodegenerative disorders.

We have developed a family of fluorescent molecular imaging probes based on 2-amino-6-acyl naphthalene element and demonstrated their in vitro capacity to label different amyloid-like neuropathological aggregates with fluorescence microscopy [1-3]. Fluorine-18 radiolabeled probe [F-18]FDDNP (2-(1-[6-[(2-[(18)F]fluoroethyl)(methyl)amino]-2-naphthyl]ethylidene) malononitrile) has been further used for determination of binding affinity to in vitro formed $\beta$ amyloid fibrils and for autoradiographic demonstration of its capacity to bind to $\beta$-amyloid plaques and hyperphosphorylated tau neurofibrillary tangles in Alzheimer's disease brain tissue slices (Agdeppa et al., 2003).

In vivo application of [F-18]FDDNP for positron emission tomography brain imaging of $\beta$ amyloid plaques and neurofibrillary tangles in Alzheimer's disease patients and subjects with mild cognitive impairment showed progression of cortical [F-18]FDDNP binding pattern ranging from medial temporal lobe labeling alone at early stages to high levels of [F-18]FDDNP binding in all cortical brain regions known to be affected by pathology in Alzheimer's type dementia [4]. This pattern agreed well with the proposed patterns at different stages of pathology evolution proposed by Braak and colleagues [5]. Increases in [F-18]FDDNP cortical binding were associated with decline in cerebral [F-18]FDG uptake, indicative of decreased cerebral glucose consumption typically observed in Alzheimer's disease.

[F-18]FDDNP has the ability to bind to fibrillar aggregates of hyperphosphorylated tau, which are the earliest pathological changes observed in Alzheimer's disease in limbic areas (hippocampus, entorhinal and transenthorinal cortex), and [F-18]FDDNP PET was successfully applied for in vivo imaging of hyperphosphorylated tau aggregates in a subset of frontotemporal dementias with neurofibrillary tangle pathology [6,7]. 
This ability distinguishes [F-18]FDNP from other $\beta$-amyloid aggregate molecular imaging probes which purportedly bind only to $\beta$-amyloid in vivo since it can be used for imaging of early stages of Alzheimer's diseases when the pathological aggregates are limited to medial temporal lobe, an area where limited binding of other amyloid molecular imaging probes has been observed even at the severe stages of Alzheimer's disease when $\beta$-amyloid pathology becomes abundant in entorhinal, rhinal and perirhinal cortices in medial temporal lobe [6,8]. Further applications of [F-18]FDDNP PET for in vivo detection of $\beta$-amyloid plaques and neurofibrillary tangles (dementia with Lewy bodies, Down syndrome), of prion plaques (Gerstmann-Straussler-Scheinker disease) and of pure hyperphosphorylated tau aggregates (a subset of frontotemporal dementia, progressive supranuclear palsy) will be discussed.

\section{References}

[1] E.D. Agdeppa et al., Mol. Imaging Biol. 5 (2003) 404.

[2] M. Bresjanac et al., J. Neurosci. 23 (2003) 8029.

[3] L.M. Smid et al., Brain Pathol. 16 (2006) 124.

[4] G.W. Small et al., N. Engl. J. Med. 355 (2006) 2652.

[5] H. Braak et al., Acta Neuropathol. Acta Neuropathol. 82 (1991) 239.

[6] J.R. Barrio et al., Acc. Chem. Res. 42 (2009) 842.

[7] N. Tolboom et al., Alzheimer Dis Assoc Disord. 24 (2010) 303.

[8] A. Kadir et al., J Nucl Med. 51 (2010) 1418.

[9] This research was supported by the US Department of Energy (DOE contract DEFG0202ER63420) and by the National Institute on Aging grant P01-AG025831. 